\title{
DIFFERENCES IN TEMOZOLAMIDE RESPONSE IN GLIOBLASTOMA MULTIFORME: A REPORT OF TWO CASES
}

\author{
Djohan Ardiansyah $^{1}$, Vita Kusuma Rahmawati ${ }^{1}$, Sri Andreani Utomo ${ }^{2}$ \\ ${ }^{1}$ Department of Neurology, Faculty of Medicine Airlangga University/Dr. Soetomo General Hospital, Surabaya, \\ Indonesia \\ ${ }^{2}$ Department of Radiology, Faculty of Medicine Airlangga University/Dr. Soetomo General Hospital, Surabaya, \\ Indonesia
}

Diterima 30 Juli 2019

Disetujui 27 Juni 2020

Publikasi 25 November 2020

Korespondensi: djo1706@yahoo.co.id
Cara merujuk artikel ini: Ardiansyah (et al). 2020.

Differences in Temozolamide Response in Glioblastoma

Multiforme: A Report of Two Cases. Callosum

Neurology Journal 3(3): 113-121. DOI:

https://doi.org/10.29342/cnj.v3i3.89

\begin{abstract}
Background: Glioblastoma multiforme (GBM) accounts for approximately $20 \%$ of primary adult-brain neoplasms and has a poor prognosis; life expectancy is 12 months or less after diagnosis. Temozolomide (TMZ) has become the standard therapy for GBM, combined with surgery and radiochemotherapy. Evaluation is important due to its association with the continuity or termination of therapy. Several studies have aimed to improve the measurement of the therapy response of GBM. The Response Assessment in Neuro-Oncology (RANO) criteria, published in 2010, has become a widely used tool for evaluating $T M Z$ response in patients with GBM. These criteria combine clinical manifestation, steroid therapy, and brain magnetic resonance imaging (MRI).
\end{abstract}

Case: We report two GBM cases with standard management of two female patients of reproductive age and with specific clinical manifestations diagnosed with
GBM in their left temporal lobes. A 24-year-old woman showed a complete response, and a 41year-old woman showed a progressive response. Discussion: We analyzed the TMZ responses for the two cases based on the RANO criteria. Prognostic factors that differentiate TMZ response in GBM include age, the Karnofsky Performance Status (KPS), the extension of surgery, presence of comorbidities, and intratumoral hemorrhage seen on MRI.

Conclusion: The differences in temozolomide response between two patients based on the RANO criteria are related to the presence of comorbidities, intratumoral hemorrhage seen on $\mathrm{MRI}$, and surgery at initial diagnosis.

Keywords: glioblastoma multiforme, prognosis, Response Assessment in Neuro-Oncology, temozolomide. 


\section{Background}

According to the World Health Organization (WHO) classification (2016), glioblastoma multiforme (GBM) is an astrocytoma grade IV tumor of the central nervous system. About $90 \%$ of GBM cases are primary gliomas arising from normal glial cells, while the remaining $10 \%$ are secondary gliomas originating from lower-grade tumors. ${ }^{1}$ This tumor accounts for approximately $20 \%$ of all adult brain tumors and is the second-largest type of tumor after meningioma. ${ }^{2}$ Epidemiological data show that GBM cases in Europe and America are affected 2-3 adults per 100,000 adults each year, with an incidence rate of men versus women is of 1.26:1. ${ }^{1}$ Life expectancy time is generally less than 12 months after diagnosis. ${ }^{3}$

The combination of surgery, radiation, and treatment with temozolomide (TMZ) has become the standard therapy for GBM. ${ }^{3,4}$ Temozolomide is also used in groups of recurrent GBM cases as a monotherapy or combination with other regimens. ${ }^{5} \mathrm{~A}$ phase III RCT study by the European Organization for Research and Treatment of Cancer (EORTC) and the National Cancer Institute of Canada - Clinical Trials Group (NCIC CTG) reported an increase in median time and life expectancy of 2 years in GBM patients treated with concomitant radiochemotherapy followed by adjuvant TMZ alone. ${ }^{6}$

The evaluation of therapy in GBM is important because it is associated with the continuation or termination of treatment. Research has been improving the ability to provide measurable evaluations in GBM management. In phase II and III trials, a study by Chinot et al. demonstrated significant measurable efficacy of therapy in GBM patients including clinical outcome, radiological response, and tumor-control duration (such as progression-free survival). ${ }^{7}$ One of the major tools for the assessment of treatment response in GBM cases is the RANO (Response Assessment in NeuroOncology) criteria introduced in 2010, replacing the MacDonald criteria (1990). ${ }^{7}$ These criteria use magnetic resonance imaging (MRI) features, clinical conditions, and steroid use as the basis for a therapeutic response. The authors reported two GBM cases with standard management and compared their TMZ responses based on the RANO criteria.

\section{Case Report}

Case 1

A 24-year-old woman, Mrs. N, presented with a major complaint of headache on the left side that had worsened over a month, blurred vision in the left eye, and projectile vomiting. No seizures, hemifacial spasms, loss of speech, hemiparesis, half-body thick sensation, fever, or history of head trauma were reported. The patient was found to be compos mentis with no meningeal signs. Focal neurologic deficits were found in the form of bilateral papilledema, right facial paresis, and mild central-right lingual paresis, as well as mild right hemiparesis.

Neuroradiologic imaging of the head using contrast MRI showed supratentorial cystic lesion, contrastenhanced in the left temporal region, of $4.81 \times 4.73 \times 4.5 \mathrm{~cm}$, accompanied by perifocal edema, a midline shift to the right as far as $0.95 \mathrm{~cm}$ and the magnetic resonance spectroscopy showed an increase in the choline to creatinine ratio. Those tended to be high-grade malignant cystic astrocytoma. The patient's KPS improved following surgery (from a score of 60 to 90). Histological examination demonstrated oval, heavy pleomorphism with multinucleated giant cell and blood vessel proliferation, mitosis, bleeding, and necrosis supporting the diagnosis of glioblastoma.
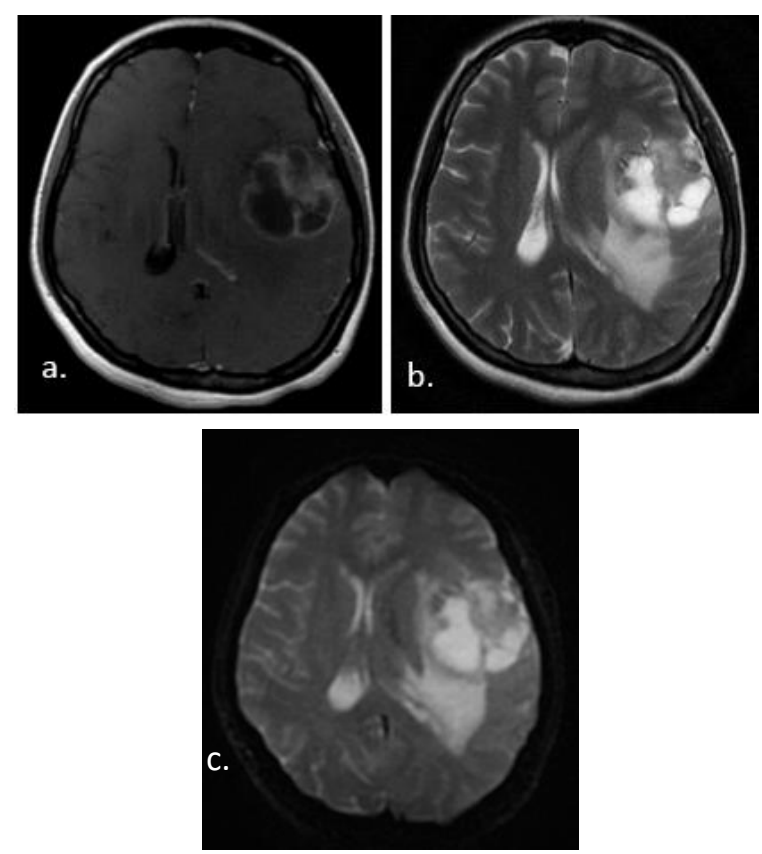

Figure 1. Initial imaging of contrast head MRI

(a) T1-weighted sequence, (b) T2-weighted sequence, (c) DWI 
CASE REPORT
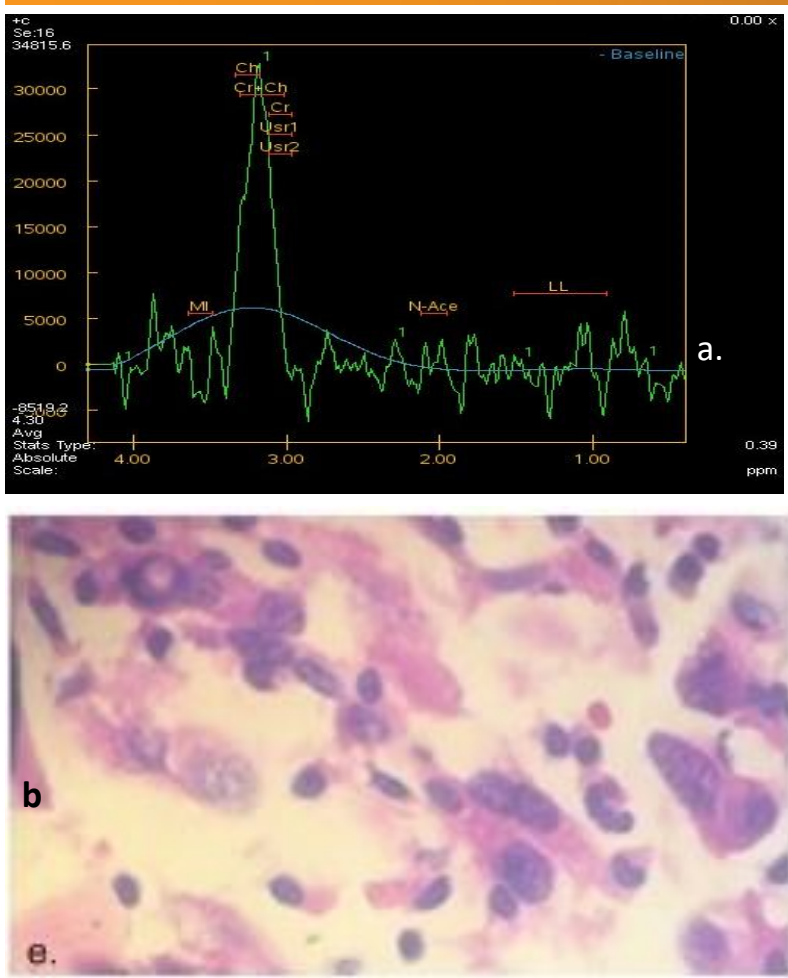

Figure 2. Initial imaging of contrast head MRI (a) MR spectroscopy, and (b) histopathological picture $^{11}$ of case 1 supporting new case of GBM.

Following surgery, the patient underwent radiochemotherapy 30 times in two months. She received a TMZ dose of $100 \mathrm{mg}$ and $0.5 \mathrm{mg}$ dexamethasone (DXM) orally once daily, simultaneously or concomitant with radiotherapy. Treatment was continued at $100 \mathrm{mg}$ per day within 7 days after concomitant therapy. Furthermore, TMZ and DXM were discontinued for 30 days. During this period, the patient's clinical condition was good, and no new neurologic deficits were found on physical examination. The imaging test (MRI) following radiochemotherapy showed minimal contrast-enhanced lesions at the surgical bed in the left temporal lobe, suspected of residual tumor or the tumor was being recurrent.

Therapy was continued with $1 \times 200 \mathrm{mg}$ adjuvant $\mathrm{TMZ}$ for six cycles, beginning four months after surgery. Each cycle comprised 5 days of $\mathrm{TMZ}$ treatment followed by a 23-day resting phase. On the second cycle, MRI showed minimal contrastenhanced lesions at the surgical bed in the left temporal lobe, suspected of being recurrent or residual post-surgical gliosis in the left temporal lobe. During this adjuvant $\mathrm{TMZ}$, she did not get steroids.
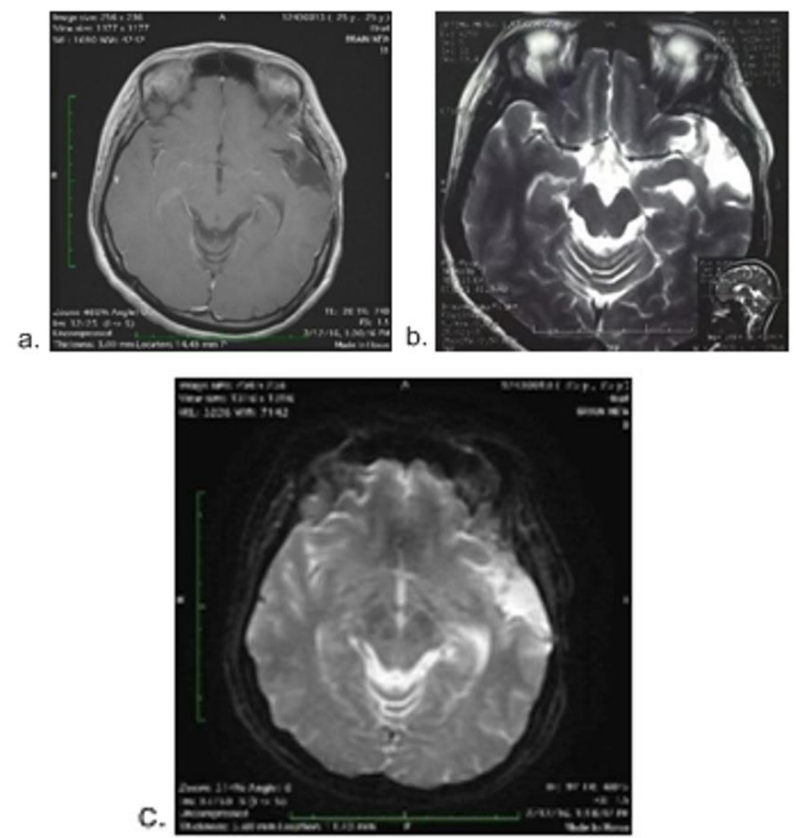

Figure 3. Case $1 \mathrm{MRI}$ imaging after radio-chemotherapy concomitant, (a) T1-weighted, (b) T2-weighted, and (c) DWI sequences.

On the fifth cycle of adjuvant TMZ, no headaches, seizures, or other complaints except for nausea, a TMZ side effect, was reported by Mrs. N. Imaging test evaluation showed no recurrent mass at the surgical bed, only an encephalomalacia cyst in the left temporoparietal region.
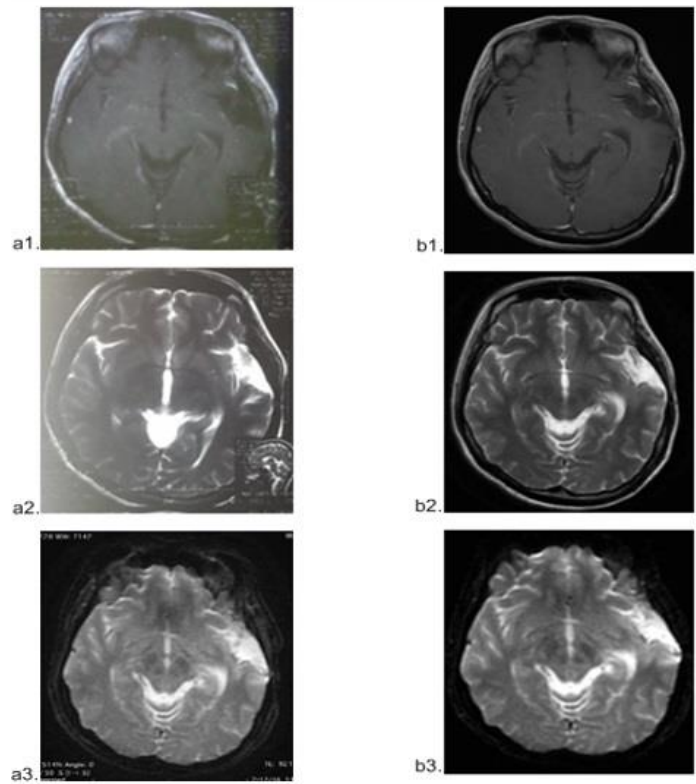

Figure 4. Comparison of contrast head MRI case 1 after adjuvant TMZ in (a) the second and (b) the fifth cycle, (1) T1-weighted, (2) T2-weighted, and (3) DWI sequences. 
Her clinical condition at the last cycle was good, with no new neurologic deficits, and a complete response was considered (KPS 100). Imaging of the head with contrast CT scan was performed and showed no features indicative of recurrent mass at the surgical bed.

\section{Case 2}

A 41-year-old woman, Mrs. G, presented with a gradual onset of speech difficulty and progressive chronic headaches. There were no complaints of hemifacial spasms, hemiparesis, hemihypesthesia, seizures, decreased consciousness, vomiting, fevers, or history of head trauma. She had type 2 diabetes mellitus as a comorbid disease, with routine insulin therapy.

General examination revealed bradypsychia and bilateral papilledema. Laboratory test results were within normal limits. Initial imaging of the head with contrast MRI showed a mass with central cystic components and a hemorrhage in the left temporal lobe, $4.22 \times 4.69 \times 4.32 \mathrm{~cm}$, accompanied by extensive tentacular edema surrounding the left lateral ventricle and ventricle III, causing a midline shift of $0.69 \mathrm{~cm}$ to the right. The magnetic resonance spectroscopy showed an increase in the choline to creatinine ratio, a decreased level of $\mathrm{N}$ acetyl aspartate (NAA), and increased lactate.

Surgery was not performed because the patient refused it. A daily dose of 60 gray radiotherapy concomitant with $1 \times 100 \mathrm{mg}$ TMZ was administered for 42 days, starting one month after onset. The patient was discharged from the hospital with clinical improvement of KPS from a score of 60 to 90. Imaging of the head with contrast MRI showed a contrast-enhanced mass with cystic components and hemorrhage in the left temporal lobe of $3.8 \times 2.4 \times 3 \mathrm{~cm}$; this was smaller than that seen in the previous MRI.

Therapy was continued with $1 \times 200 \mathrm{mg}$ adjuvant TMZ for 7 cycles, starting 3 months after onset. Contrast MRI of the head following TMZ showed a contrast-enhanced mass of $4.32 \times 3.5 \times 2.35 \mathrm{~cm}$ in the left temporal lobe. The size was relatively the same compared to that seen in the previous MRI. Hence, TMZ was continued for up to 12 cycles. The patient had focal seizures in the 11th cycle, and clinical examination showed central-right facial and lingual palsy. The brain contrast MRI test after the entire $T M Z$ cycle revealed a mass of $5.29 \times 4.2 \times 3.37 \mathrm{~cm}$ with a necrotic area in the left temporal lobe, with surrounding edema. A larger tumor was found compared to the previous MRI, accompanied by displacement of the left lateral ventricle to the right. This condition supported a diagnosis of recurrent GBM. Tumor excision and craniotomy were conducted followed by radiochemotherapy and adjuvant TMZ. The histopathology test of tumor excision showed tissue with extensive necrosis and proliferation of round to oval, pleomorphic heavy nucleated anaplastic cells confirming GBM.

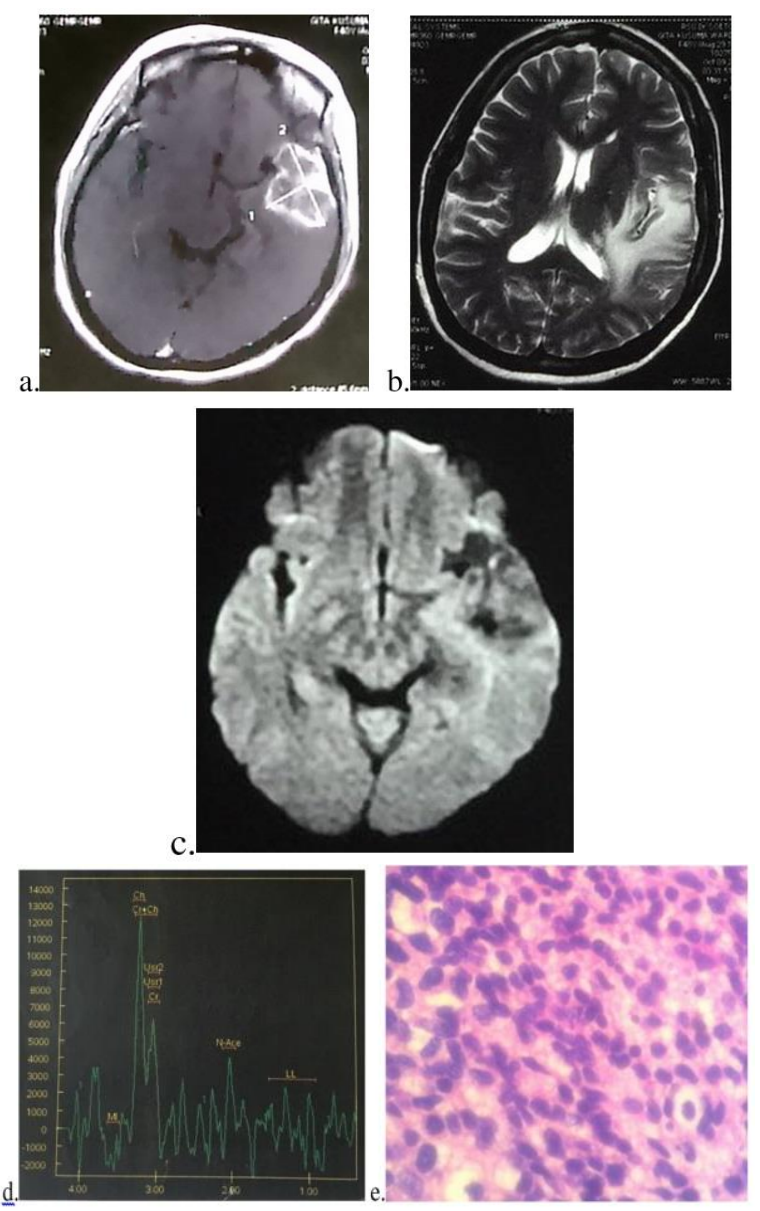

Figure 5. Initial Contrast head MRI of case 2, (a) T1weighted, (b) T2-weighted, (c) DWI sequences, (d) MR spectroscopy, and

(e) histopathological picture ${ }^{11}$ after surgery confirming GBM.

Concomitant radiochemotherapy with 60 grays and $1 \times 100 \mathrm{mg}$ TMZ was conducted 18 months after onset. Contrast head MRI showed a GBM mass of $3.6 \times 3.3 \times 4.3 \mathrm{~cm}$; it was relatively more fixed than previously seen and accompanied by noncommunicating hydrocephalus and an increase in surrounding edema. 

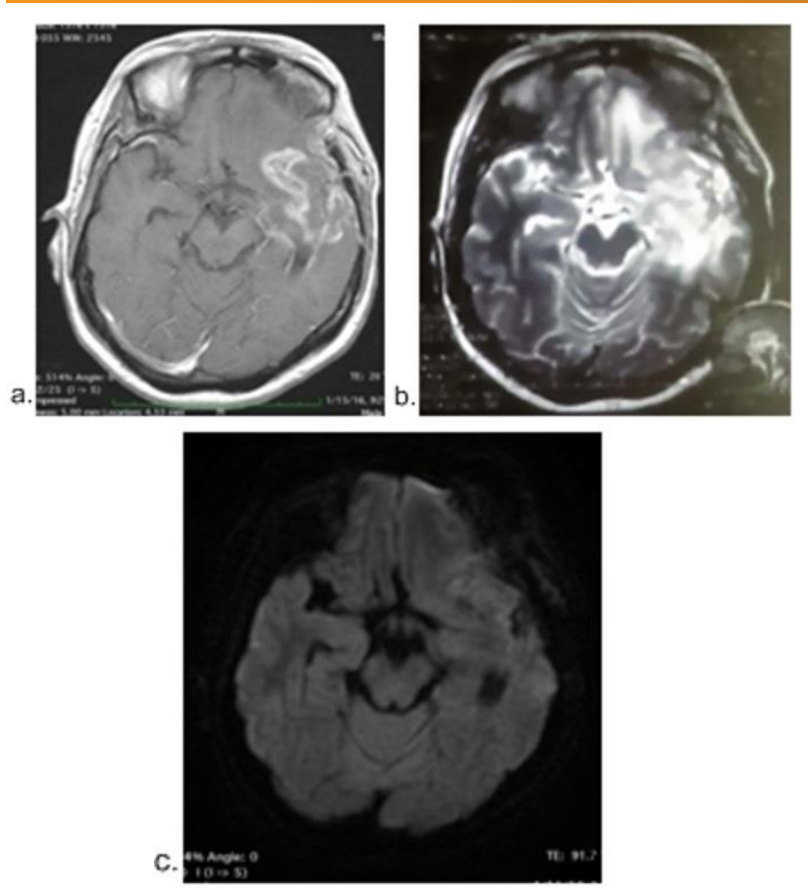

Figure 6. Case 2 contrast MRI after surgery and concomitant radio-chemotherapy, (a) T1-weighted, (b) T2-weighted, (c) and DWI sequences.

After a month, adjuvant TMZ with $1 \times 200 \mathrm{mg} \mathrm{TMZ}$ was administered for 5 days, with a 23-day resting phase. The dosage of DXM was tapered after two weeks in each cycle of therapy. The imaging test on the second cycle showed an intra-axial mass in the left temporal horn with a necrotic area and extensive perifocal edema resulting in a $1.4-\mathrm{cm}$ midline shift to the right. The patient was found to have several neurologic deficits during these periods but improved clinically with steroid administration and rehabilitation therapy. The brain contrast MRI after the fifth cycle showed an intra-axial mass in the cortical and subcortical regions of the left temporal lobe of $5 \times 4,4 \times 5,4 \mathrm{~cm}$ with a necrotic area and perifocal edema pressing on the left lateral ventricle. The size of the mass was larger in comparison to that shown by the previous MRI. The patient's clinical condition became progressively worse, and she died 26 months after being diagnosed with GBM.

\section{Discussion}

Both case reports showed GBM in women in their reproductive age, and 1.5 times more frequent in men than in women, and twice as frequent in people with lighter skin coloring. ${ }^{9}$ Weingart et al. also found a greater prevalence of case data in men than in women, approximately 1.6:1.0, and that onset was more likely to appear during the sixth decade. Although GBM has rarely been found in African Americans, there is no significant difference in the incidence of GBM cases after adjusting for race and age. ${ }^{10}$
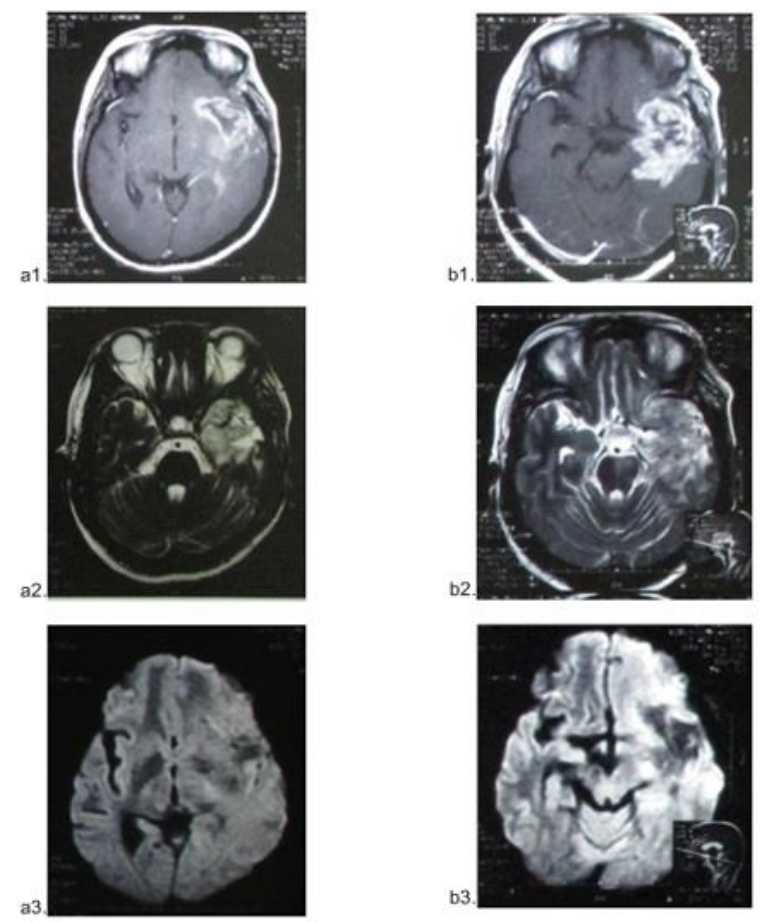

Figure 7. Comparison of contrast head MRI case 2 after repeated adjuvant $\mathrm{TMZ}$ in (a) the second and (b) the fifth cycle, (1) T1-weighted, (2) T2weighted, and (3) DWI sequences.

Clinical symptoms of GBM can be either specific or nonspecific. Case 1 shows a nonspecific major complaint of gradually increasing severe left-side headaches, whereas case 2 shows gradually increasing severity of difficulty talking and progressive chronic headaches. Headaches occur in about $77 \%$ of patients with GBM and comprise $40 \%$ of early symptoms related to malignant gliomas. Its pattern is intermittent, sharp, and pressing, and they frequently occur in the morning, worsening as the day progresses and/ or with physical activity. Headache in the morning appears as a result of hypercapnia during sleep and ends in cerebral vasodilation and continued headache. The location of the headache is often difficult to determine, but pressure on the meninges structure of the headache area may indicate the location of the tumor. ${ }^{11}$

Besides, both cases showed specific early symptoms. In the first case, a focal neurologic deficit of the central-right facial and lingual paresis and mild right hemiparesis were found. In the second case, language disorder was found. These 
specific symptoms may indicate the location of lesions or may be a consequence of the effect of the mass. In $42 \%$ of patients, hemiparesis was noted, while $14 \%$ showed hemianesthesia. ${ }^{10}$ Lesions are often located in the cerebral hemispheres and rarely in the posterior fossa and brainstem. ${ }^{11}$

The rapid development of neuroradiology has made it easier for practitioners to find new cases of GBM and evaluate therapy. In terms of the radiological location of the tumor, both cases presented in the present study show the same location in the left temporal lobe. Specific and nonspecific clinical symptoms can include the effects of a tumor mass on surrounding brain parenchyma. In a study by Tanaka et al. (2013) of 105 patients with GBM, 60 patients or $57 \%$ had a characteristic tumor site in the temporal lobe. ${ }^{2}$

Both cases showed classic features of GBM on imaging of contrast head MRI. A T1-weighted sequence showed an intra-axial mass tumor with a contrast-enhanced ring and a central necrotic area. A contrast-enhanced ring border is indicative of an active tumor. The features of T2-weighted MRI in both cases also revealed a hyperintense area around the lesions called perilesional edema, which indicates infiltrative tumors. The second case showed more heterogeneous mass features and intratumoral bleeding. The DW1 sequences showed a non-restricted diffusion area. These three sequences strongly support the existence of a GBM, adjusted to clinical conditions. Spectroscopic MR revealed increased choline and creatinine as well as lactate but a relative decrease in NAA. These features correspond to high-grade glioma neoplasms. A ratio of twice as much choline as creatinine strongly supports the presence of a high-grade glioma. ${ }^{14}$

The initial standard management for GBM is surgery, followed by concomitant radiochemotherapy and adjuvant TMZ therapy. In case 1 , surgery was performed immediately after head MRI, whereas case 2 involved concomitant radiochemotherapy due to the patient's refusal of surgery. Surgery in case 2 was performed when new neurologic deficits were found in the only adjuvant TMZ based on MRI, which showed an increased size in tumor mass and extension of perilesional edema, reflecting a recurrence of GBM. Adjuvant TMZ therapy was performed after concomitant radiochemotherapy in both cases. In case 1 , the patient received adjuvant TMZ for 6 cycles after concomitant radiochemotherapy with prior surgery. In case 2 , the patient received adjuvant $T M Z$ twice; the first adjuvant $T M Z$ was performed after concomitant radiochemotherapy without prior surgery, while the second was performed following standard management, i.e. after concomitant radiochemotherapy with prior surgery. The timing for the adjuvant TMZ therapy was also different; the first was for 12 cycles and the second for 5 cycles. Adding adjuvant TMZ after radiochemotherapy is clinically and statistically significant and provides a survival benefit with minimal toxicity. ${ }^{4}$ Optimal dosage and usage duration of $\mathrm{TMZ}$ are still subjects of debate. ${ }^{5}$

The evaluation of adjuvant TMZ in GBM is highly significant. Several criteria were examined in this evaluation. ${ }^{11} \mathrm{~A}$ study by Chinot et al. applied the RANO criteria to evaluate the $T M Z$ response in GBM. The evaluations included complete response, partial response, stable disease, and progression. Imaging of contrast T1, contrast T2/FLAIR, or DWI sequence; patient's clinical condition and use of steroids form the basis of a TMZ response. It is recommended that the RANO criteria be applied within 12 weeks after radiochemotherapy. Based on the RANO Working Group, these criteria also be applied post-surgery, radiosurgery, and immunotherapy. ${ }^{7}$

The TMZ response after radiochemotherapy is analyzed by comparing the differences in the MRI features of the early and final cycles of the adjuvant TMZ. Steroid use and clinical condition are also analyzed in the RANO criteria. We assessed the adjuvant $T M Z$ response after the patient received concomitant radiochemotherapy and surgery. The first case involved 6 cycles of adjuvant TMZ. The patient complained only of nausea as a side effect of TMZ and was not given until the last cycle. These three criteria - radiologic features no existence of contrast enhancement and new lesions, good clinical conditions, and no steroid use - are included in the complete response according to the RANO criteria.

The patient underwent two adjuvant $\mathrm{TMZ}$ treatments in the second case. The second adjuvant TMZ was performed after surgery and radiochemotherapy, with DXM for two weeks in each cycle of adjuvant TMZ. Although the steroid dosage was increased, the patient's clinical condition continued to worsen. In consideration of the clear severity of a patient's clinical condition, even if the steroid dosage was increased and a significantly increasing lesion size was noted based on $\mathrm{MRI}$, the $\mathrm{TMZ}$ response included in the progression criteria shows recurrence. Postponing surgery as initial therapy of standard GBM 
management has the potential to decrease overall survival time.

The patient in case 2 also suffered from type 2 diabetes mellitus. In a retrospective cohort study by Chambless et al. (2012) of 171 patients with high- grade gliomas, 15 patients $(8.8 \%)$ had a history of diabetes mellitus type 2 . In these patients, there was a decrease in median overall survival (312 and 470 days, $p=0.003$ ) and Progressive Free Survival (PFS) (106 and 166 days, $p=0.04$ ) compared to non-diabetic patients. ${ }^{12}$ Early $\mathrm{MRI}$ in case 2 showed intratumoral bleeding and extensive perifocal edema, describing the infiltrative characteristic of the tumor. There may also have been $\mathrm{TMZ}$ resistance. A review article by Lee (2016) found that $50 \%$ of GBM patients treated with $\mathrm{TMZ}$ failed to respond to it. ${ }^{13}$ In a review, Repeated resections are considered for progressive or recurrent GBM to improve the patient's life expectancy and to confirm the diagnosis, primarily histologically. The average survival time is 14 weeks. ${ }^{14}$

In both cases, the prognostic factors differentiating adjuvant $\mathrm{TMZ}$ response after concomitant radiochemotherapy and surgery according to the RANO criteria are as follows: the presence of comorbidity, intratumoral bleeding shown on MRI before diagnosis, and surgery as the initial standard treatment for GBM management. Several studies investigated the effectiveness of $T M Z$ in standard GBM management. A study by Jeon (2009) of 79 new GBM cases in patients with a median age of 52 years provided primary overall survival output data. The median overall survival time was 18.3 months ( $95 \% \mathrm{Cl}$; $16.3-20.1$ months), and the rate of progression was 6.7 months (95\% $\mathrm{Cl}$; 5.2-8.3 months). Survival rates for year 1 and year 2 were $70.1 \%$ and $37.1 \%$, respectively. More than 80 patients with KPS after operation show survival rate longer compared to patients with KPS values lower than 80 (23.1 months compared to 13.4 months, $p<0.001) .{ }^{15}$ A study by Lee (2012) demonstrated that radiochemotherapy and adjuvant TMZ in older patients with GBM resulted in longer survival rates. Management of radiochemotherapy and adjuvant $\mathrm{TMZ}$ administered to 70 patients older than 70 years diagnosed with GBM was examined. The median overall survival time was 11.8 months $(95 \% \mathrm{Cl}$; $8.7-$ 14.8). Based on multivariate analysis, total tumor excision is significantly related to longer overall survival time (hazard ratio $[\mathrm{HR}]=0 ; 236 ; 95 \% \mathrm{Cl}$; $0.060-0.922 ; p=0.038$ ) and good functional status
(HR=0.124; 95\% Cl; 0.022-0.693; $\mathrm{p}=0.017) .{ }^{16}$ In another study, 25 adult patients with a median age of 53 years diagnosed with GBM and with KPS scores higher than 70 were prospectively studied with a median follow-up of 12.4 months. All patients underwent radiochemotherapy, and 19 patients (76.0\%) underwent adjuvant TMZ. The median overall survival time was 15.67 months (95\% Cl; 11.56-20.04). The delay of radiotherapy up to more than 6 weeks following surgery is an independent prognostic factor related to reduced overall survival time (4.0 compared to 16.1 months; $p=0.027) .{ }^{17}$

The Karnofsky Performance Status scale is a general measurement to determine the prognosis of GBM based on the clinical condition. Several factors were related to increased survival time of GBM patients; these included higher KPS values post-surgery $(p=0.0001)$, radiation therapy $(p<0.0001)$, younger age $(p=0.0443)$, and the nonexistence of diabetes $(p=0.0006) .{ }^{18}$

Furthermore, age is an important factor in GBM prognosis. Older age at onset is reported to be a significantly disadvantageous prognostic factor for GBM. Non-aggressive treatment is considered for older GBM patients, such as biopsy or palliative care due to the potential for intolerance to standard therapies. ${ }^{2}$ The percentage of patients less than 40 years old with a survival rate within 5 years for $34 \%$ compared to only $65 \%$ of those aged years 40 or older. The age of 40 years is the threshold in the group division of patients with GBM related to disease prognosis. ${ }^{19} \mathrm{~A}$ study by Darefsky et al. (2012)

showed that a group of 20-44 years old with GBM had a survival time of five years. In the cases diagnosed with GBM in this age group, the survival rate is 5 years in $12 \%(95 \% \mathrm{Cl}, 9 \%-16 \%)$ in 19931995 and $16 \%$ (95\% Cl, 13\%-20\%) in 2002-2004. In GBM patients in this group who underwent surgery and radiotherapy, the survival rate in five years is $14 \%(95 \% \mathrm{Cl}, 10 \%-20 \%)$ in cases diagnosed from $1993-1995$ and $19 \%(95 \% \mathrm{Cl}, 15 \%-23 \%){ }^{20}$

\section{Conclusion}

Prognostic factors that differentiate $\mathrm{TMZ}$ response in both cases were the presence of comorbidity, intratumoral hemorrhage seen on MRI, and surgery performed early in the diagnosis. Based on the RANO criteria and prognostic factors that support a $\mathrm{TMZ}$ response, the role of adjuvant $\mathrm{TMZ}$ can be said to be important in the standard management of GBM. 
References

1. Urbańska K, Sokołowska J, Szmidt M and Sysa P. Glioblastoma multiforme-an overview. Contemp Oncol (Pozn). 2014; 18: 307-12. https://www.ncbi.nlm.nih.gov/pubmed/2547 7751. Accessed June 27, 2019.

2. Tanaka S, Meyer FB, Buckner JC, Uhm JH, Yan ES and Parney IF. Presentation, management, and outcome of newly diagnosed glioblastoma in elderly patients: clinical article. Journal of neurosurgery. 2013; 118: 786-98.

https://www.ncbi.nlm.nih.gov/pubmed?cmd= Link\&dbFrom=PubMed\&from_uid=25768833. Accessed June 27, 2019.

3. Szczepanek D, Marchel A, Moskała M, Krupa $M$, Kunert $P$ and Trojanowski T. Efficacy of concomitant and adjuvant temozolomide in glioblastoma treatment. A multicentre randomized study. Neurologia $i$ neurochirurgia polska. 2013; 47: 101-8. http://www.neurology-

asia.org/articles/neuroasia-2017-22(1)049.pdf. Accessed June 27, 2019.

4. Stupp R, Mason WP, Van Den Bent MJ, et al. Radiotherapy plus concomitant and adjuvant temozolomide for glioblastoma. New England Journal of Medicine. 2005; 352: 987-96. https://www.nejm.org/doi/full/10.1056/NEJ Moa043330. Accessed June 28, 2019.

5. Khasraw M, Bell D and Wheeler H. Long-term use of temozolomide: could you use temozolomide safely for life in gliomas? Journal of clinical neuroscience. 2009; 16: 854 5.

https://www.ncbi.nlm.nih.gov/pubmed/1930 3779. Accessed June 28, 2019.

6. Stupp R, Hegi ME, Mason WP, et al. Effects of radiotherapy with concomitant and adjuvant temozolomide versus radiotherapy alone on survival in glioblastoma in a randomised phase III study: 5-year analysis of the EORTCNCIC trial. The lancet oncology. 2009; 10: 45966.

https://www.ncbi.nlm.nih.gov/pubmed/1926 9895. Accessed June 29, 2019.

7. Chinot OL, Macdonald DR, Abrey LE, Zahlmann G, Kerloëguen $Y$ and Cloughesy TF. Response assessment criteria for glioblastoma: practical adaptation and implementation in clinical trials of antiangiogenic therapy. Current neurology and neuroscience reports. 2013; 13: 1-11. https://www.ncbi.nlm.nih.gov/pubmed/2352 9375. Accessed June 27, 2019.

8. Ernawati E. Perbaikan Klinis dan Harapan Hidup pada Pasien Glioblastoma Multiforme (GBM): Laporan Tiga Kasus. Aksona. 2016; 1: 6-14.

9. Alifieris $\mathrm{C}$ and Trafalis DT. Glioblastoma multiforme: Pathogenesis and treatment. Pharmacology \& therapeutics. 2015; 152: 6382.

https://www.ncbi.nlm.nih.gov/pubmed/2594 4528. Accessed June 29, 2019.

10. Weingart JD, McGirt MJ and Brem H. HighGrade astrocytoma/glioblastoma. Oncology of CNS tumors. Springer, 2010, p. 147-61.

11. Ardiansyah D. Glioma: Diagnose Step, Treatment, and Follow Up. In: Suharjanti IB, Mudjiani; and Islamiyah, WR (ed.). Rational Management in Neurological Practice. Surabaya: Dep/ SMF Neurologi Fakultas Kedokteran Unair/ RSUD Dr Soetomo, Surabaya; Dwiputra Pustaka Jaya, 2016, p. 273-85.

12. Chambless LB, Parker SL, Hassam-Malani L, McGirt MJ and Thompson RC. Type 2 diabetes mellitus and obesity are independent risk factors for poor outcome in patients with high-grade glioma. Journal of neuro-oncology. 2012; 106: 383-9. https://www.ncbi.nlm.nih.gov/pubmed/2183 3800. Accessed June 29, 2019.

13. Lee SY. Temozolomide resistance in glioblastoma multiforme. Genes \& Diseases. 2016;3(3):198-210.

https://www.ncbi.nlm.nih.gov/pubmed/3025 8889. Accessed June 29, 2019.

14. Franceschi E, Tosoni A, Bartolini S, Mazzocchi V, Fioravanti A and Brandes AA. Treatment options for recurrent glioblastoma: pitfalls and future trends. Expert review of anticancer therapy. 2009; 9: 613-9. https://www.ncbi.nlm.nih.gov/pubmed/1944 5578. Accessed June 29, 2019.

15. Jeon HJ, Kong DS, Park KB, et al. Clinical outcome of concomitant chemoradiotherapy followed by adjuvant temozolomide therapy for glioblastomas: single-center experience. Clinical neurology and neurosurgery. 2009; 111:

679-82. https://www.ncbi.nlm.nih.gov/pubmed/1964 0635. Accessed June 27, 2019.

16. Lee J-H, Jung T-Y, Jung $S$, et al. Performance status during and after radiotherapy plus concomitant and adjuvant temozolomide in 
elderly patients with glioblastoma multiforme. Journal of Clinical Neuroscience. 2013; 20: 503-8. https://www.jocnjournal.com/article/S0967-5868(12)00451-

1/abstract. Accessed June 29, 2019.

17. Jastaniyah $\mathrm{N}$, Murtha $\mathrm{A}$, Pervez $\mathrm{N}$, et al. Phase I study of hypofractionated intensity modulated radiation therapy with concurrent and adjuvant temozolomide in patients with glioblastoma multiforme. Radiation oncology. 2013; 8:

https://rojournal.biomedcentral.com/articles/ 10.1186/1748-717X-8-38. Accessed June 29, 2019.

18. Chambless LB, Kistka HM, Parker SL, HassamMalani L, McGirt MJ and Thompson RC. The relative value of postoperative versus preoperative Karnofsky Performance Scale scores as a predictor of survival after surgical resection of glioblastoma multiforme. Journal of neuro-oncology. 2015; 121: 359-64. https://www.ncbi.nlm.nih.gov/pubmed/2534 4883. Accessed June 29, 2019.

19. Walid MS. Prognostic factors for long-term survival after glioblastoma. Perm J. 2008; 12: 45-

8.https://www.ncbi.nlm.nih.gov/pubmed/213 39920. Accessed June 29, 2019.

20. Darefsky AS, King JT and Dubrow R. Adult glioblastoma multiforme survival in the temozolomide era: A population-based analysis of Surveillance, Epidemiology, and End Results registries. Cancer. 2012; 118: 2163-72.

https://www.ncbi.nlm.nih.gov/pubmed/2188 2183. Accessed June 29, 2019. 